\title{
Normally Unused Positional Cues Guide Ectopic Afferents in the Leech CNS
}

\author{
M. Beatrice Passani, Alejandro Peinado, ${ }^{a}$ Holly Engelman, Carlos A. Baptista, ${ }^{b}$ and Eduardo R. Macagno \\ Department of Biological Sciences, Columbia University, New York, New York 10027
}

\begin{abstract}
Central projections from peripheral sensory neurons segregate into distinct, ventrally positioned longitudinal tracts within the segmental ganglia of the leech Hirudo medicinalis. As documented here, there is an additional tract in the neuropils of the fifth and sixth body ganglia, located at the lateral margin and formed by afferent axons (the "sex afferents") originating from sensory neurons located in the male and female genitalia. Ablation of the genitalia results in the complete absence of this additional tract.

We asked (1) whether segmental differences exist in the distribution of pathway cues available to the sex afferents, and (2) whether central pathway selection by these axons is specific. We transplanted the primordia of the male genItalia to several ectopic positions posterior to the sixth body segment and labeled the ectopic sex afferents in order to examine their paths in the CNS. In about $50 \%$ of the experimental animals, afferent axons originating in the transplanted tissue segregated into a distinct lateral fiber bundle within the neuropil of a nearby ganglion, in a position corresponding to their normal one in the sex ganglia. The sex afferents therefore find their normal pathways even in the ganglia of inappropriate segments, although these pathways are not used by any other afferents in these ganglia.

We propose, therefore, that the positional cues employed by afferent axons to select appropriate pathways in the ganglionic neuropil are expressed in all segments of the leech CNS, regardless of whether such cues are normally used by afferent axons in each segment.
\end{abstract}

Are the guidance cues used by afferent projections distributed widely throughout the CNS, or are they restricted to well-defined locations? This is an important issue in the study of the development of neuronal projections. Observations in many systems have shown that axons generally take highly reproducible pathways (e.g., Ghysen, 1978; Raper et al., 1983), and have provided the basis for the hypothesis that pathways are labeled with cues that can be recognized by axonal growth cones as they navigate

\footnotetext{
Received Apr. 30, 1991; revised July 2, 1991; accepted July 17, 1991.

We thank Dr. Birgit Zipser for the gift of the Lan3-2 antibody, Dr. Laura Wolszon for her critical reading of the manuscript, Michael Nitabach for help in the preparation of back-filled nerves, and Nicholas Necles for help with the photography. This work was supported by grants from the National Institutes of Health and the National Science Foundation.

Correspondence should be addressed to Eduardo R. Macagno, Department of Biological Sciences, Columbia University, 1003B Fairchild, New York, NY 10027.

- Present address: Department of Neurobiology, Duke University Medical Center, Durham, NC 27710.

' Present address: Department of Pathology, College of Physicians and Surgeons, Columbia University, New York, NY 10032.

Copyright (C 1991 Society for Neuroscience 0270-6474/91/113868-09\$05.00/0
}

toward their targets (reviewed in Dodd and Jessell, 1988). Such cues are generally thought to be utilized by the earliest axons growing along a route, which have been called "leaders" (LoPresti et al., 1973) or "pioneers" (Bate, 1976). Later-growing axons may use the same cues or, alternatively, use a different mechanism to recognize and fasciculate with earlier-growing axons.

The search for pathway markers has met with some success in the periphery. Investigations of the genesis of afferent projections in the insect limb bud, for example, have led to the identification of guidepost cells that appear to specify the route taken by pioneer axons (Bentley and Keshishian, 1982). However, other cues may also be important and part of a hierarchy of choices available to these growth cones (Berlot and Goodman, 1984).

Within the CNS, advances have been made in the identification of molecules expressed by particular axons or even regions of axons (Bastiani et al., 1987; Dodd et al., 1988). However, the distribution of those molecules that have been characterized is suggestive of their having a role in the selective fasciculation of fibers, rather than in the initial establishment of the pathways.

One way to ascertain whether the cues used by particular axons are more widely distributed than would be surmised from an examination of normal axonal routes has been to confront axons with a novel region of the nervous system and observe how they respond. For afferent axons, this approach is equivalent to posing the following question: can a group of sensory axons growing ectopically make a "normal" projection in a region of the CNS that they do not usually occupy and, more specifically, into a region of neuropil that does not normally receive a sensory projection? The answer to this question could shed some light not only on the development of the nervous system, but also on the evolution of neural structures. For example, it is possible that particular cues, previously required for axon deployment, continue to be expressed after evolutionary changes in the structure of the organism have eliminated the need for those particular cues.

Previous studies in invertebrates involving either transplantation of sensory neurons to ectopic locations or homeotic mutations that transform entire appendages (reviewed in Anderson, 1981; Palka, 1982; see also Murphey et al., 1983, 1985), as well as studies of the projections of ectopic eyes in amphibia (Constantine-Paton and Capranica, 1976; Constantine-Paton, 1978; Katz and Lasek, 1979), have demonstrated that cues used by ingrowing afferents are repeated in the CNS at different loci along the anteroposterior axis. From these studies, however, it has not been possible to ascertain whether such cues are present in locations other than those where they are normally used by some subset of sensory projections. In contrast, transplantation of the acousticolateral placode to the position of the eye results 
in the formation of an ectopic VIIIth nerve that follows a normally unused trajectory in the diencephalon (Constantine-Paton, 1983). This result suggests that pathway cues may be generally available in the neuraxis.

In the leech Hirudo medicinalis, as documented here, there is a unique central projection of peripheral sensory neurons located in the genitalia. It is present in only 2 of the 21 segmental ganglia (SG) of the body and occupies a location in the neuropil distinct from that of all other sensory afferents. We have taken advantage of this unique projection in the leech to probe for the existence of positional cues within the CNS, in places where such cues appear not to be required for the development of normal sensory afferent projections.

\section{Materials and Methods}

Animals. Embryos of the leech Hirudo medicinalis obtained from our colony were maintained at $23^{\circ} \mathrm{C}$. Embryos were removed from their cocoons at 9-10 d of embryogenesis, at which time the transplantations were performed. Thereafter, they were kept in sterile artificial spring water (Instant Ocean, Menasha Corporation; $0.5 \mathrm{gm} / \mathrm{liter}$ ).

Surgical procedures. The following operations were carried out in 9or 10-d-old embryos: homotopic or heterotopic transplantation of the male organs, attached to the body wall, or heterotopic transplants of patches of skin and body wall. The male genitalia are located in the fifth body segment and comprise the penis, prostate gland, epididymes, and ejaculatory ducts. In the embryo, the primordia of these organs are located slightly posterior to segmental ganglion 5 (SG5).

The transplants were carried out as previously described (Baptista and Macagno, 1988). Briefly, embryos were anesthetized in 9\% ethanol in sterile leech Ringer's, which is composed of $116 \mathrm{~mm} \mathrm{NaCl}, 4 \mathrm{~mm}$ $\mathrm{KCl}, 1.8 \mathrm{~mm} \mathrm{CaCl}_{2}$, and $10 \mathrm{~mm}$ Tris maleate, $\mathrm{pH} \mathrm{7.4.} \mathrm{Two} \mathrm{embryos} \mathrm{of}$ the same age were placed ventral side up and side by side, in grooves cut into a Petri dish coated with Sylgard (Dow Corning Corp., Midland, MI). The male genitalia and some of the surrounding tissue were removed from the donor embryo and transplanted into an opening of adequate size in the skin of the host embryo; the normal male genitalia of the host animal were left in place. In most cases, we found that the afferents from the transplant innervated the nearest ganglion. Successful transplants were made in the fifth $(n=6)$, seventh $(n=9)$, tenth $(n=$ $20)$, twelfth $(n=5)$, and fifteenth $(n=4)$ body segments. In a control group $(n=10)$, we transplanted a patch of skin, instead of the male organs, to either the tenth $(n=4)$, the twelfth $(n=3)$, or the fifteenth $(n=3)$ body segment.

After allowing a short period for wound healing, the grafted animals were returned to sterile artificial spring water and allowed to develop to a postembryonic stage (older than $30 \mathrm{~d}$ ). In most cases, the ectopic male reproductive tissue developed normally; the penis, prostate, and epididymes appeared to be of approximately normal size. Branches of the ganglionic nerve roots were seen to innervate the ectopic tissue on one or both sides in many cases. In others, nerves to the transplanted tissue were not visible, but it is possible that they were missed because of their being very fine. In any case, the presence of visible innervation was not a prerequisite for finding a sex afferent projection in the CNS, and, in a few cases, afferents from the reproductive tissues were absent from the CNS despite the clear presence of a nerve branch innervating them.

Labeling of afferent fibers with monoclonal antibodies. Postembryonic animals were dissected after being anesthetized in $9 \%$ ethanol in leech saline for 10-20 min. Ganglia were removed, pinned in a Sylgard-coated dish, fixed for $1 \mathrm{hr}$ in $4 \%$ paraformaldehyde (in $0.1 \mathrm{~m}$ phosphate buffer, $\mathrm{pH}$ 7.4), dehydrated in an ethanol series, and processed for immunostaining either in whole-mount or in cross sections. To cut transverse sections, fixed ganglia were embedded in polyethylene glycol (PEG; Sigma), $80 \% 3350 \mathrm{MW}$ polymer and $20 \% 1450 \mathrm{MW}$ polymer. The infiltration was done at $60^{\circ} \mathrm{C}$ with three 20 min changes in $50 \%$ PEG in ethanol followed by three 20 min changes in 100\% PEG. Transverse sections were cut at a thickness of $1.8 \mu \mathrm{m}$ and collected on gelatincoated glass slides. Sections were incubated in the supernatant of monoclonal antibody Lan3-2 [1:2 in 0.01 M phosphate-buffered saline (PBS) and $2 \%$ normal goat serum] for $\mathrm{l} \mathrm{hr}$. The antibodies were visualized with either rhodamine-conjugated goat anti-mouse (Boehringer Mann- heim; 1:50 for $1 \mathrm{hr}$ ) or with biotin-conjugated secondary antibodies (1: 50 in PBS for $1 \mathrm{hr}$ ) and amino-methyl coumarin acetic acid (AMCA)conjugated avidin (3:100 in $50 \mathrm{~mm}$ HEPES buffer, $\mathrm{pH} 8.2$; Vector Laboratories) for $1 \mathrm{hr}$. Tissue sections were coverslipped with a watersoluble nonfluorescent mounting medium (polyvinyl alcohol, glycerol, and PBS) and photographed with a Leitz microscope equipped for epifluorescence.

Whole-mount preparations were incubated in collagenase $(2000 \mathrm{U} / \mathrm{ml}$; Sigma type IV) prior to fixation, to allow better penetration of the antibodies through the sheath surrounding the nervous system. After fixing them as above for $1 \mathrm{hr}$ and rinsing in PBS, the tissue was incubated overnight $\left(4^{\circ} \mathrm{C}\right)$ in the supernatant of the monoclonal antibody Lan3-2 (1:4 in PBS containing 1\% Triton X-100). Anti-Lan3-2 labeling of segmental ganglia was visualized with peroxidase-antiperoxidase secondary antibodies (1:50 in PBS plus 1\% Triton X-100), whereas immunoreactivity in the male reproductive tissue was visualized with avidin-conjugated Texas red. Male organ whole-mount preparations were also stained with Hoechst 33258 (Polyscience; $0.5 \mathrm{~mm}$ in PBS) to confirm the presence of sensory cell bodies in the penile sheath.

Anterograde labeling of afferent fibers. Two techniques were used to determine the sites of origin of the afferents fasciculating in the lateral afferent tract. For controls, the animal was opened along the dorsal side and the sex nerves from SG6 to the male reproductive tissue were disconnected from the male organ and dipped in a 30\% solution of rhodamine dextran-amine (RDA; Molecular Probes, Eugene, OR) in sterile Ringer's for $2 \mathrm{~d}$. This technique could not be used for experimental animals, since the ectopic sex nerves were too short to be manipulated. Instead, RDA crystals were inserted with a fine needle into the ectopic male gonopore of animals that had been anesthetized in 15 mM chlorobutanol (Sigma) dissolved in artificial spring water. After a few minutes, animals were returned to artificial spring water and the dye was allowed to diffuse for about $2-3 \mathrm{~d}$ in order to label mainly afferents, and longer times if the retrograde labeling of central neurons was desired as well. Ganglia were then dissected and processed for antibody staining in cross sections, as described above.

\section{Results}

Most SG of the Hirudo medicinalis ventral nerve cord are structurally very similar to one another, with the exception of the two "sex" ganglia, SG5 and SG6, which innervate the male and female genitalia and are specialized for reproductive functions. All ganglia receive afferent projections from sensory neurons located in the skin, body wall, and internal organs. Afferents fasciculate with one another as they travel centripetally along peripheral nerves and enter the SG through the paired lateral root nerves. Afferent projections can be visualized either by anterograde filling with a dye, such as RDA, or with the monoclonal antibody Lan3-2 (Zipser and McKay, 1981), which recognizes surface antigens common to all known peripheral sensory neurons in Hirudo, regardless of their sensory modalities (Peinado, 1988; Peinado et al., 1990). The known exception is a putative stretch receptor located in the body wall (the Hoover cell; Blackshaw et al., 1984), which has a much larger axon than other peripheral sensory neurons and does not stain with Lan3-2 (Peinado, 1988).

\section{The fifth and sixth body segmental ganglia have an additional afferent tract}

As reported previously (Peinado et al., 1987a,b), staining with Lan3-2 reveals that, after entering the ganglionic neuropil, afferent projections turn anteriorly and posteriorly and segregate into ipsilateral longitudinal tracts that run in the ventral half of the neuropil. These tracts continue beyond the neuropil and extend to adjacent ganglia along the interganglionic connectives (schematized in Fig. 1; see also Fig. 2A,B).

We have now found an additional, Lan3-2-positive longitudinal tract in the neuropils of the sex ganglia, SG5 and SG6. This additional tract occupies a lateral position, at the margin 

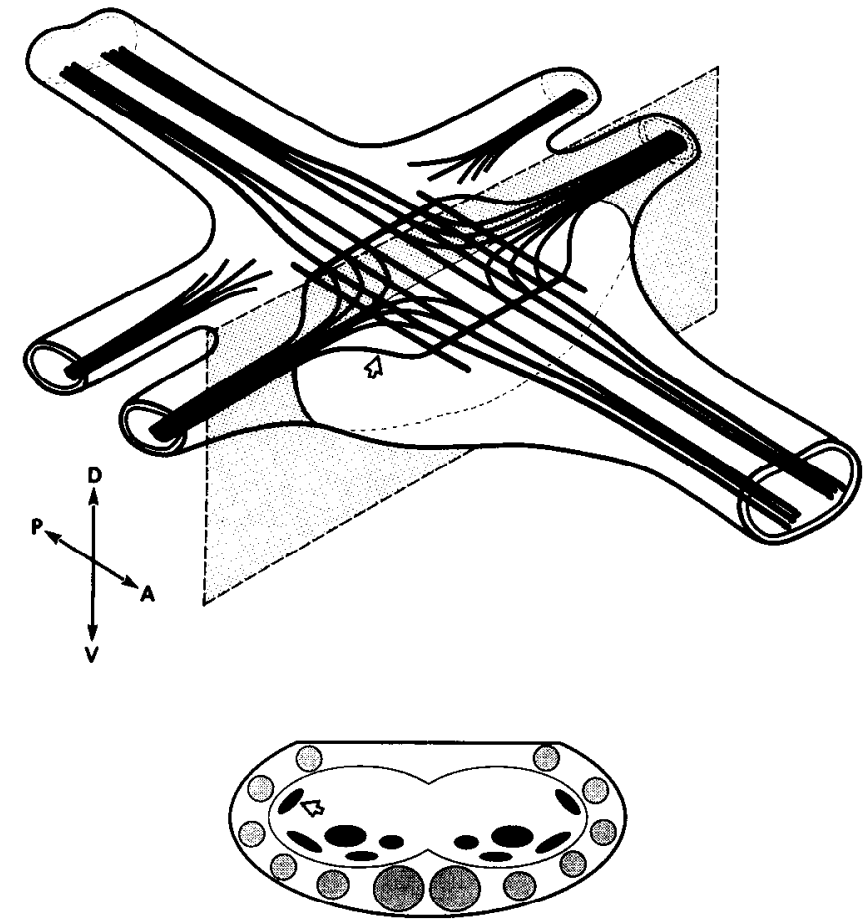

Figure 1. Schematic drawings showing the afferent tracts in a sex ganglion. The upper drawing shows a tilted view of the ganglion in the orientation indicated by the directional arrows at left $(D$, dorsal; $V$, ventral; $A$, anterior; $P$, posterior). Bundles of afferent axons are shown entering the ganglion through the anterior and posterior nerve roots and then joining the afferent tracts in the neuropil (those from the posterior roots are cut short for the sake of clarity). The longitudinal afferent tracts common to all ganglia are shown traveling ventrally through the ganglion and into the interganglionic connective nerves. The sex afferent projections (indicated on one side by the open arrow) are unique to the sex ganglia; afferent axons are confined to the neuropil of these ganglia where they form lateral tracts, and decussate through both anterior and posterior commissures. The shaded plane indicates the position of the cross section shown in the lower drawing. In this diagram, neuronal somata are shown as shaded circles surrounding the neuropil, and the tracts are indicated by solid ellipses. The open arrow indicates one of the sex afferent tracts. (These drawings are based on preparations like those shown in Fig. 2.)

of the neuropil (Fig. 2D1,D2). We noted as well another unique feature of the sex ganglia, the projection of some afferent axons to the contralateral side of the neuropil through anterior and posterior commissures (Fig. 2C; see also schematic in Fig. 1).

The results of two types of experiments demonstrated that the additional tract originated in cells specifically associated with the sex organs. First, anterograde labeling of the sex afferent axons with a dye, such as RDA, either by dipping the sex nerves in a highly concentrated solution or by applying crystals of the dye to the male gonopore, revealed clearly stained axons in the sex tract of both SG5 and SG6 (Fig. 2D2). Labeled fibers originating in the male organ entered the neuropil through the anterior nerve roots. Some of them turned anteriorly and followed the lateral tract and then turned again to project contralaterally; others projected into two of the ventral tracts after entering the neuropil and did not decussate. A small fraction projected posteriorly and decussated approximately at the level of the posterior roots (Fig. 2C). These anterogradely labeled axons also showed Lan3-2 immunoreactivity in the neuropil (Fig. 2DI) and in the male organ (Fig. 3).
Table 1. Projection patterns in homotopic and heterotopic transplants (no. of cases)

\begin{tabular}{lclll}
$\begin{array}{l}\text { SG inner- } \\
\text { vated after } \\
\text { transplant }\end{array}$ & $\begin{array}{l}\text { Presence of sex } \\
\text { afferent tract }\end{array}$ & $\begin{array}{l}\text { Bilateral } \\
\text { projection }\end{array}$ & $\begin{array}{l}\text { Unilateral } \\
\text { projection }\end{array}$ & $\begin{array}{l}\text { Decus- } \\
\text { sating } \\
\text { fibers }\end{array}$ \\
\hline SG5 ${ }^{a}$ & $6(n=6)$ & 2 & 4 & 6 \\
SG7 & $1(n=9)$ & 0 & 1 & 0 \\
SG10 & $11(n=20)$ & 2 & 9 & 3 \\
SG12 & $4(n=5)$ & 1 & 3 & 0 \\
SG15 & $1(n=4)$ & 1 & 0 & 0
\end{tabular}

$n$, Number of surviving animals with transplants.

${ }^{a}$ Homotopic transplant; all others heterotopic.

Anterograde labeling by placing dye in the female gonopore resulted in the staining of significantly fewer afferents (not shown). These innervated only SG6, through its posterior nerve roots, and appeared to travel in the same tracts as the afferents from the male gonopore.

The second line of evidence indicating that these afferents originatc in genital tissucs is that animals that had their male and female sexual organs removed surgically at $9-10 \mathrm{~d}$ of development lacked the dorsolateral tract and commissural fibers altogether (Fig. 4B). Removal of only the male organs resulted in a complete lack of these afferent pathways in SG5 and a significant reduction in SG6.

\section{Sex afferents from transplanted male genitalia can form ectopic lateral tracts}

To determine whether segmental differences exist in the neuropilar cues available to afferent fibers, we transplanted primordia of male genitalia (along with a small amount of body wall and skin) from donor animals to host segments posterior to segment 6 ("heterotopic" transplants). To control for possible effects of the surgery, primordia from a donor were placed into the fifth segment of some hosts, following the removal of their original genitalia ("homotopic" transplants). Donors and hosts were embryos 9 or $10 \mathrm{~d}$ old, an age during which the nervous system is still undergoing extensive differentiation (Macagno et al., 1990). About $50 \%$ of the operated animals survived to adulthood and were analyzed in these experiments. Table 1 summarizes the number of transplants done in various segments and the results obtained.

Ectopic transplants were successfully integrated into the ventral body wall in 44 animals. In most of these, it was possible to identify, during dissection, at least one branch of a ganglionic nerve root that appeared to have innervated the transplanted male tissue. In all 44 cases, the ganglion associated with or closest to the ectopic genitalia was sectioned transversely and stained with Lan3-2. Twenty-three of these non-sex ganglia (52\%) showed novel afferent tracts in the lateral positions corresponding to the sex tracts of SG5 and SG6 (cf. Fig. $4 A$ and $4 C I$ ). To confirm that these afferents originated in the transplanted genitalia, 11 of the experimental animals first had a crystal of RDA placed in the transplanted gonopore, and the nearby host ganglia were examined for the presence of RDA-labeled afferents. In seven of these ganglia, labeled axons were found in the lateral location appropriate for the sex afferents (Fig. 4C2), with some axons joining two of the other tracts, as is normally the case in the sex ganglia. In the remaining four cases, RDA-labeled afferents were found only in the common afferent tracts. 

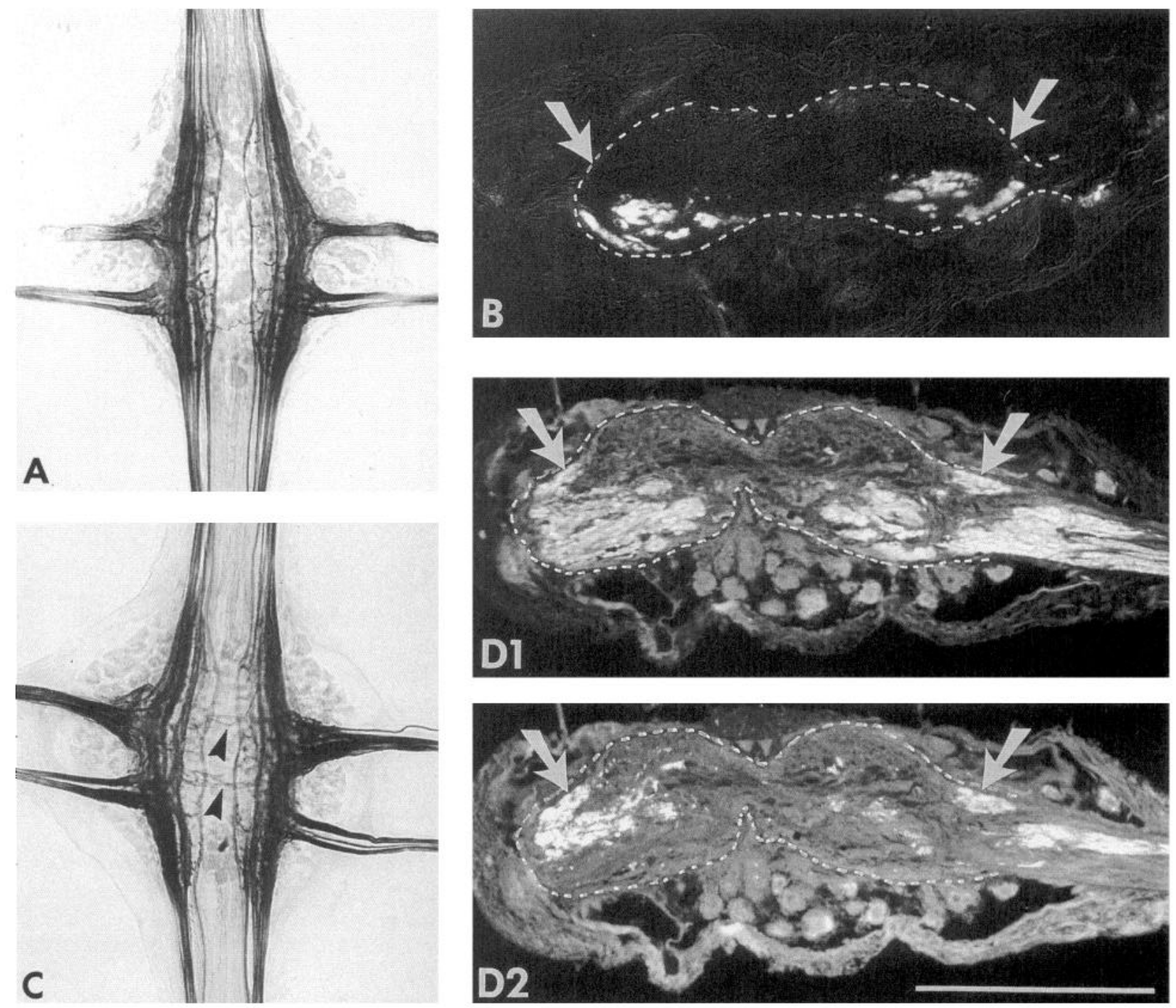

Figure 2. Central tracts of axons from peripheral sensory neurons in non-sex and sex ganglia, as revealed by immunostaining with the monoclonal antibody Lan 3-2 or by dye filling with RDA. The Lan3-2 antibody was visualized with a peroxidase-antiperoxidase secondary antibody in the whole-mount preparations and with AMCA-conjugated avidin in the transverse sections. $A$, Whole-mount preparation of a non-sex ganglion, showing sensory afferents sorting into bilateral tracts oriented longitudinally within the neuropil. At these magnifications, individual axons cannot be distinguished in the labeled bundles. In $A$ and $C$, anterior is up. $B$, Transverse section of a non-sex ganglion stained with Lan3-2. Note that the immunostained afferent tracts are localized in the ventral half of the neuropil and that there is no immunostaining at the position of the lateral pathway (location indicated by arrows; compare with $D 1$ ). Here and D1 and D2, dorsal is up and the broken line indicates the margin of the neuropil. $C$. Whole-mount of a sex ganglion (SG6) immunostained with Lan3-2. Note that some stained afferents project across the midline of the ganglion, through anterior and posterior commissures (arrowheads). D1, Transverse section from another SG6, immunostained with Lan3-2. The additional pair of tracts in lateral positions in the neuropil (arrows) are those specific for sex afferents originating in the genitalia. Stained afferents can be seen entering the ganglion on the right side; the plane of section is roughly in the same location as the plane indicated in Figure 1, but cuts through the anterior root on this side. Endogenous fluorescence reveals neuronal somata surrounding the neuropil (demarkated with a broken line), particularly on the ventral side (bottom) of the ganglion. D2, Same section as in D1, but photographed through filters that reveal axons filled with RDA. To label the sex afferents, the sex nerves that innervate the male genitalia were dipped in an RDA solution prior to fixation and sectioning. This resulted in the labeling of afferent as well as efferent axons. The sex afferents in the lateral tracts are indicated by the arrows; other RDA-filled afferents can be seen in the ventral tracts stained with Lan3-2 (compare with D1). RDA-filled efferents (arrowheads) lack Lan3-2 immunostaining (compare with $D 1$ ). Scale bar in $D 2: 250 \mu \mathrm{m}$ for $A$ and $C ; 50 \mu \mathrm{m}$ for $B ; 75 \mu \mathrm{m}$ for $D 1$ and $D 2$.

It is important to note that thorough examination of normal animals, by anterograde labeling of afferents with dye placed in peripheral tissues or nerves, and by staining with a variety of antibodies specific for subsets of afferent projections in Hirudo (Peinado et al., 1987b), failed to show any evidence of afferents in this lateral position in ganglia other than the sex ganglia (e.g., Fig. $2 B$ ). Therefore, our data suggest that, although these pathways are normally unused in non-sex ganglia, markers for the sex afferents must still exist in these particular regions, allowing the formation of ectopic sex tracts.

The success rate in forming the lateral projections in ganglia innervating an ectopic male organ differed significantly among the locations tried (Table 1). These projections were found in 1 out of 4 cases in SG15, in 4 out of 5 cases in SG12, in 11 out of 20 cases in SG10, and in 1 case out of 9 in SG7. We do not know at present the basis for this positional variability. 

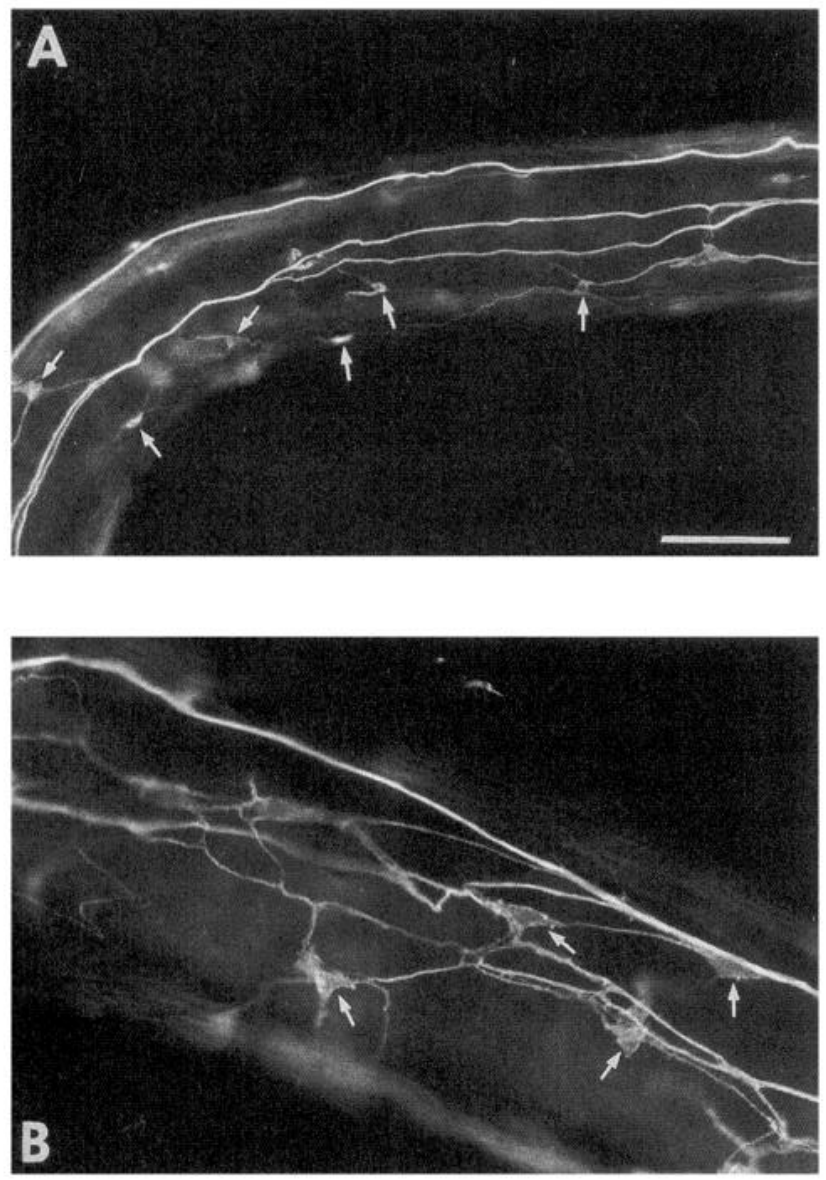

Figure 3. Lan3-2 immunostaining of sensory neurons in the male organ. $A$, Whole mount preparation showing Lan3-2-immunopositive cells in the penile sheath. Double staining with Hoechst 33258 (not shown) revealed nuclei within the cell bodies marked with arrows. $B$, Magnification of another region of the preparation in A. Arrows indicate cell bodies. Scale bar: $100 \mu \mathrm{m}$ for $A ; 50 \mu \mathrm{m}$ for $B$.

There were also differences in the extent to which transplantderived afferents reproduced normal projection patterns (Fig. 5). For example, ectopic sex afferents usually failed to project to the contralateral neuropil; we found decussating sex afferents only in 3 of the 11 cases with ectopic projections in SG10, and none in the other successful heterotopic transplants. Also, the size of the lateral tract, while varying considerably, was always smaller than that of its counterpart in normal sex ganglia. A likely possibility is that a fraction of the fibers simply failed to reach their new target, although it is also possible that they were misrouted and fasciculated with fibers in the other afferent tracts. Consistent with the second possibility is the observation that in four cases where sex afferents had been stained with both RDA and Lan3-2, doubly labeled axons were found only in the ventral tracts (not shown).

The transplant-derived afferent projections also differed from the normal pattern in some other respects. Whereas the normal projection is always bilateral, there were only four ganglia with heterotopic transplants in which Lan3-2 revealed sex afferent tracts on both sides of the neuropil (Table 1). We assume that this reflected the formation of a unilateral nerve to the transplanted organ; in the majority of cases, it was not possible to see such nerves during dissection because the transplant was placed very close to the ganglion in order to facilitate innervation. Moreover, while male sex afferents normally enter the sex ganglia through the anterior roots, the transplanted afferents did so through either the anterior or posterior nerve roots, or through both. Processes entering along the posterior roots usually turned posteriorly and extended in this direction farther than they normally do. Nevertheless, the sex afferent projection always took a lateral position in the neuropil, regardless of the place of entry or the longitudinal direction in which the fibers eventually projected. The diagrams presented in Figure 5 illustrate schematically the variety of patterns of ectopic afferents that were found in these experiments, along with the normal pattern in the sex ganglia for comparison.

The results of the control homotopic transplantations showed that the surgical procedures may have been partly responsible for the apparent inability of heterotopically transplanted fibers to generate a completely normal afferent projection. Only two of the six experimental animals with a transplant in segment 5 had bilateral sex afferents, but in all six cases we observed fibers decussating at positions comparable to those normally followed by these afferents. Thus, transplantation per se does seem to affect the ability of the CNS to innervate the transplanted tissues bilaterally, but it does not appear to render afferents unable to cross the midline. The relatively small percentage of instances of decussating sex afferents in heterotopic transplants (3 of 17 cases, or $17.6 \%$ ), in comparison to $100 \%$ (6 out of 6 cases) for homotopic transplants, could well reflect segmental variations in the presence of appropriate guidance cues in the ganglionic commissures.

To control for the possibility that transplantation might itself induce non-sex afferents to form a lateral tract, we transplanted patches of ventral skin and body wall to segments 10,12 , or 15 in embryos 9 or $10 \mathrm{~d}$ old. None of the 10 specimens, examined by staining transverse sections with Lan3-2, contained such a tract (not shown). In every case, afferents generated projections that were restricted to the ventral neuropil in a manner comparable to that for normal afferents in these ganglia.

\section{Discussion}

\section{Sex afferents can reproduce normal central projections} ectopically

In the normal adult leech, only the two sex ganglia receive afferent projections from sensory neurons in the male and female genitalia. Within the corresponding neuropils, most of these axons travel in specific longitudinal afferent tracts to reach their central targets. We assume that many of the sex afferents establish the special lateral tract by responding to one set of guidance cues, while the rest recognize other cues to join other afferents in two of the ventral tracts common to all ganglia.

Since the ventral tracts containing multimodal sensory axons are present in all ganglia, and some sex afferents normally join these tracts in the sex ganglia, it was not surprising to find, while examining animals with ectopic sex organs, that a subgroup of the transplanted sex afferents were able to join these tracts ectopically. This subgroup may use the same pathway cues as do other afferents in these tracts or may recognize the other afferents themselves and fasciculate with them. In fact, this result is much like that obtained in several other systems. In insects, for example, studies of homeotic sensory afferents in Drosophila mutants (reviewed in Palka and Ghysen, 1982) and of ectopic afferent projections derived from transplants in fly (Schmid et al., 1986), locust (Anderson and Bacon, 1979; Bacon and Anderson, 

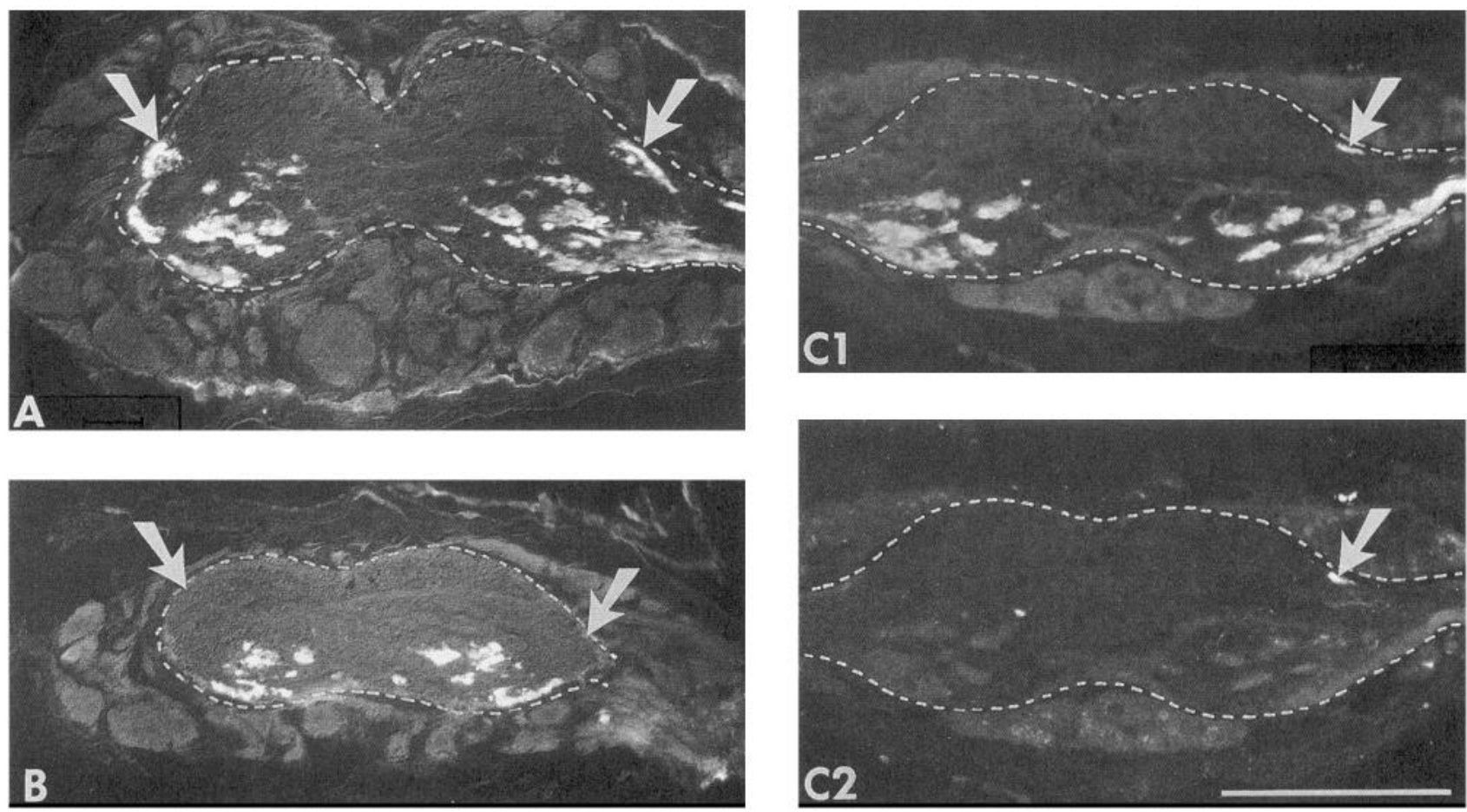

Figure 4. Transverse sections from normal $(A)$ and experimental ganglia ( $B$ and $C$ ). The broken line indicates the margin of the neuropil. $A$, Lan32 -immunostained afferents in a normal SG6, shown here for the purpose of comparison with the results shown in the other panels. The arrows indicate the lateral sex afferent tracts. $B$, The lateral sex afferent tracts are absent in SG6 from an adult animal that had its male and female genitalia removed during embryogenesis. The arrows indicate the positions normally occupied by the lateral sex afferent tracts (compare with $A$ ). $C l$ and $C 2$, Double labeling of a section of SG10 from an adult animal that had male genitalia transplanted to the tenth body segment during embryogenesis. $C 1$ shows the afferent tracts immunostained with Lan3-2 and visualized with AMCA-conjugated avidin. The ectopic sex afferents indicated by the arrow segregate in a lateral position that is comparable to the lateral sex afferent tract seen in SG5 and SG6 (compare with $A$ ). $C 2$ is the same section as in $C l$ but shows the rhodamine fluorescence in the RDA-filled ectopic sex afferent axons segregating in the lateral tract (arrow). In this, as in most animals with ectopic transplants, we found the ectopic sex afferents entering the neuropil from only one side (see Table 1). Dorsal is up. Scale bar, $50 \mu \mathrm{m}$.

1985), and cricket (Murphey et al., 1983, 1985) have also considered the behavior of axons growing into abnormal central locations. These misplaced afferents were generally found either to project into tracts normally used by other afferents, or to follow pathways pioneered by their segmental homologs. Similarly, following early eye transplantation in amphibia, ectopic optic axons travel in pathways that parallel local sensory or other tracts, and hence may be following other axons rather than reading specific but normally unused cues (Constantine-Paton, 1983). For example, displaced optic axons entering the neuraxis at various levels form abnormal longitudinal projections that are consistently located laterally in the spinal cord (ConstantinePaton and Capranica, 1976; Katz and Lasek, 1979; Harris, 1980). It is possible that they do so by recognizing cues distributed throughout the nervous system, but it is also possible that they are growing along the ascending or descending sensory tracts found in this region, a possibility that has not, to our knowledge, been tested directly by doubly labeling the native and displaced axons. Recent observations on the distribution of antigens recognized by monoclonal antibodies raised against visual system determinants (Takagi et al., 1987), however, lend some support to the alternative explanation, that guidance cues used by optic axons are widely distributed.

Our principal finding strongly suggests that there is a wide distribution of axonal pathway markers in the developing leech nervous system. Transplanted sex afferents can establish their special lateral projection in more posterior ganglia, where no afferent axons of any kind normally project. We interpret this as evidence that the markers of this pathway are present in segments where they are never used by afferent projections during normal development. A very similar conclusion was reached
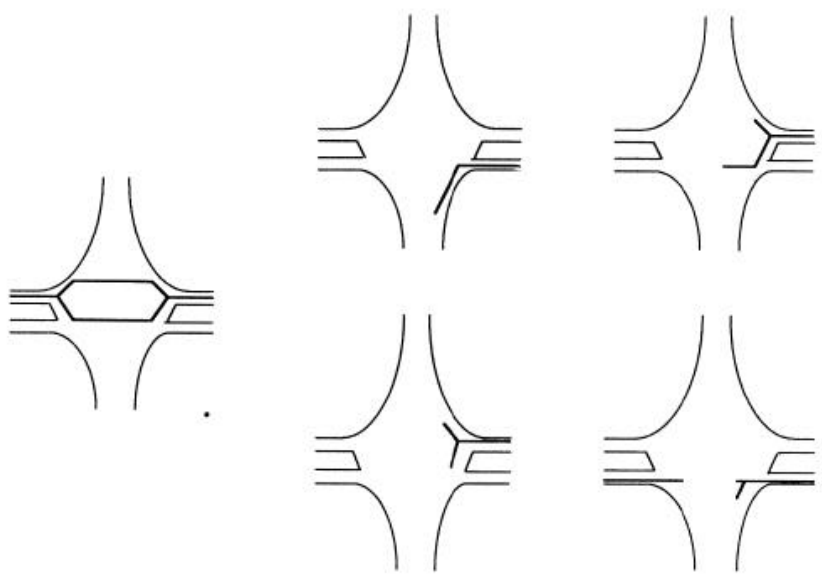

A

B

Figure 5. Ectopic sex afferents show a variety of projection patterns in host neuropils. The schematic diagrams show the normal projection pattern generated in the sex ganglia $(A)$ and four examples of ectopic sex afferent projections as reconstructed from serial transverse sections stained with Lan3-2 (B). Anterior is up. 
by Constantine-Paton (1983) on the basis of the results of embryonic transplantation of the acousticolateral placode to a vacated eye position in the frog Rana pipiens (Constantine-Paton, 1983). In $50 \%$ of the operated animals, Constantine-Paton observed an ectopic VIIIth nerve that entered the diencephalon laterally and coursed dorsally, taking a trajectory not normally followed by any other axons in this location, but closely resembling the normal path taken by the VIIIth nerve at the level of the medulla. She concluded that the most likely explanation for this result was that the structural elements used by these axons for guidance were widely distributed in the neural tube, even in locations where they are never used in normal development.

\section{What might be the nature of the lateral pathway cues?}

The markers that axons utilize to grow to their targets include (1) gradients of attractive factors (e.g., Lumsden and Davies, 1983; Tessier-Lavigne et al., 1988; Heffner et al., 1990), (2) specific markers recognized only by particular growing axons (Bastiani et al., 1986; Yamamoto et al., 1986), and (3) general positional markers that can be interpreted by many, if not all, axons (Bozyczko and Horwitz, 1986; Cohen et al., 1987; Bronner-Fraser, 1988; Letourneau et al., 1988; Tomaselli et al., 1988). Spatially fixed markers could reside on extracellular elements or on the surfaces of epithelial, glial, or neuronal cells.

Judging by the relative distances involved, it seems unlikely that gradients of chemotropic agents direct sex afferents to the lateral pathway, although this possibility cannot, in principle, be dismissed "out of hand." Leech afferents generally do grow toward the CNS, and they might do so under the influence of chemotropic factors released by it. However, once the axons enter the ganglionic neuropils, they consistently turn anteriorly and posteriorly to form tracts that are only a few micrometers apart (Fig. 1), a behavior that would be hard to explain on the basis of individual diffusible chemoattractants. We think it more likely, therefore, that the displaced afferents (and, for that matter, all afferent fibers) use stable guidance information localized to the position of the appropriate afferent tracts.

The above explanation is also supported by other current work in the leech and in other systems (e.g., Bastiani et al., 1987; Patel et al., 1987; reviewed in Dodd and Jessell, 1988). In the leech, specific subsets of afferent projections have been shown to express different $130 \mathrm{kDa}$ glycoproteins on their surfaces (Flaster et al., 1983; Peinado et al., 1990). These molecules could be involved in pathway recognition, as well as in selective fasciculation or defasciculation of afferent axons (Zipser et al., 1989). A glycoprotein unique to the sex afferents of the lateral tract has not been identificd thus far, however.

Another possible source of pathway markers in the leech CNS are glial cells, as suggested in other systems (e.g., Jacobs and Goodman, 1989a,b; reviewed in Hatten and Mason, 1986). Despite the lack of direct evidence of such a role for leech glia in development, their locations (Cole et al., 1989) and molecular heterogeneity (Flaster and Zipser, 1987) suggest that they should be considered as a possible source of positional cues. However, it should be noted that the ablation of interganglionic connective glia does not affect the accurate regeneration of adult axons, suggesting that these glia, at least, do not have a role in axon guidance in the adult (Elliott and Muller, 1983a,b).

Other likely candidates for providing pathway markers are the central targets of afferent projections. For example, interneurons that receive synaptic inputs from the sex afferents might travel longitudinally throughout the nerve cord and be present in all ganglia. Little is currently known about what the central targets of the sex afferents are, but there is some indirect evidence that the Retzius ( $\mathrm{Rz}$ ) cells and the rostral penile evertor motor neurons in the sex ganglia are among them. Peinado (1988) has shown that these cells in the sex ganglia extend dendrites into the region of the lateral tract, whereas the homologs of the $\mathrm{Rz}$ cells in other ganglia do not. Interestingly, the $\mathrm{Rz}$ cells in the sex ganglia are morphologically different from those in other segments, but the latter can become like the former if they contact male reproductive tissue transplanted to ectopic locations (Loer and Kristan, 1989). Whether this transformation includes sending branches to the vicinity of the sex afferent tract has not yet been ascertained, but if so, these branches could be a source of the pathway markers. This explanation appears to raise the additional question of what directs the $\mathrm{Rz}$ cell branches into the position of the lateral tract but does not invalidate the hypothesis that all SG have the same potential of guiding afferent fibers to the appropriate central location, regardless of whether this is specified by cues always available or induced by the presence of the male organ primordium. In this regard, it is worth noting that profound effects of sensory projections upon central structures have been documented in other systems. One example is found in Manduca sexta, where male-specific afferents from a male antenna transplanted to a female host during pupal stages induce the formation of a male-specific olfactory glomerulus not normally present in the female brain (Schneiderman et al., 1982). Another example is in the mouse, where displaced olfactory axons induce the ectopic formation of structures resembling glomeruli following neonatal bulbectomy (Graziadei and Monti Graziadei, 1986).

\section{Why are pathway markers expressed where they are not needed?}

A general trend in the evolution of metameric animals is toward segmental specialization. It is possible, therefore, that in some ancestral stage there may have been a sex afferent tract in more segments than those where it is presently found. For example, more segments may have been involved in reproductive functions in leech ancestors (see discussion in Sawyer, 1986) or, perhaps, the lateral afferent pathway might have been used by other types of afferents that have been lost or diverted to one of the other tracts. The cues that guide the growth of ectopic sex afferents to the lateral pathway in non-sex ganglia of Hirudo might have been preserved because they play a more general role during CNS development than we could ascertain by examining only the formation of afferent projections. Some light could also be shed on these possibilities by examining pathway organization and usage in other annelids.

However, the reason these markers are expressed in nonsex segments could lie in a different direction, in the form, for instance, of a general requirement of the ontogenetic program. It is well known from the work of Stent, Weisblat, and colleagues (Weisblat et al., 1980; Stent and Weisblat, 1985; Torrence and Stuart, 1986) that early development is very similar in all segments of the leech. Setting up the afferent tracts is also an early event (McKay et al., 1984; Stewart et al., 1985; Zipser et al., 1989), repeated in all body segments, and thus is probably an invariant part of the general developmental program. Therefore, generating the markers in every segment may be simpler than modifying this program to accommodate the requirement of the sex afferents only in the SG5 and SG6. This and other ways in which nonadaptive determinants might have guided the evo- 
lution of neural circuits have becn discussed by Dumont and Robertson (1986).

In summary, our observations indicate that axon guidance cues capable of supporting the generation of precise patterns of neuronal projections are considerably more widespread than might be surmised from an analysis of normal neural development and anatomy. As in other systems, the molecular mechanisms having this function in the leech remain to be characterized.

\section{References}

Anderson H (1981) Projections from sensory neurons developing at ectopic sites in insects. J Embryol Exp Morphol 65:209-224.

Anderson H, Bacon J (1979) Developmental determination of neuronal projection patterns from wind-sensitive hairs in the locust, Schistocerca gregaria. Dev Biol 72:364-373.

Bacon J, Anderson H (1985) Developmental determination of central connections from wind-sensitive hairs in the locust, Schistocerca gregaria. J Exp Biol 114:679-684.

Baptista CA, Macagno ER (1988) The role of the sexual organs in the generation of postembryonic neurons in the leech Hirudo medicinalis. J Neurobiol 19:707-726.

Bastiani MJ, du Lac S, Goodman CS (1986) Guidance of neuronal growth cones in the grasshopper embryo. I. Recognition of a specific axonal pathway by the pCC neuron. J Neurosci 6:3518-3531.

Bastiani MJ, Harrelson AL, Snow PM, Goodman CS (1987) Expression of fasciclin I and II glycoproteins in subsets of axon pathways during neuron development in the grasshopper. Cell 48:745-755.

Bate CM (1976) Pioneer neurones in an insect embryo. Nature 260: 54-56.

Bentley D, Keshishian H (1982) Pathfinding by peripheral pioneer neurons in grasshopper. Science 218:1082-1088.

Berlot J, Goodman CS (1984) Guidance of peripheral pioneer neurons in the grasshopper: adhesive hierarchy of epithelial and neuronal surfaces. Science 223:493-495.

Blackshaw S, McKay DA, Thompson SWN (1984) The fine structure of a leech stretch receptor neuron and its efferent supply. J Physiol (Lond) 350:76P.

Bozyczko D, Horwitz AF (1986) The participation of a putative cell surface receptor for laminin and fibronectin in peripheral neurite extension. J Neurusci 6:1241-1251.

Bronner-Fraser M, Lallier T (1988) A monoclonal antibody against a laminin-heparan sulfate proteoglycan complex perturbs cranial neural crest migration in vivo. J Cell Biol 106:1321-1329.

Cohen J, Burne JF, McKinley C, Winter J (1987) The role of laminin and the laminin/fibronectin receptor complex in the outgrowth of retinal ganglion cells. Dev Biol 122:407-418.

Cole RN, Morell RJ, Zipser B (1989) Glial processes, identified through their glial-specific $130 \mathrm{kD}$ surface glycoprotein, are juxtaposed to sites of neurogenesis in the leech germinal plate. Glia 2:446-457.

Constantine-Paton M (1978) Central projections of anuran optic nerves penetrating hindbrain or spinal cord regions of the neural tube. Brain Res 158:31-43.

Constantine-Paton M (1983) Trajectories of axons in ectopic VIIIth nerves. Dev Biol 97:239-244.

Constantine-Paton M, Capranica RR (1976) Axonal guidance of developing optic nerves in the frog. I. Anatomy of the projection from transplanted eye primordia. J Comp Neurol 170:17-32.

Dodd J, Jessell TM (1988) Axon guidance and the patterning of neuronal projections in vertebrates. Science 242:692-699.

Dodd J, Morton SB, Karagogeos D, Yamamoto M, Jessell TM (1988) Spatial regulation of axonal glycoprotein expression on subsets of embryonic spinal neurons. Neuron 1:105-116.

Dumont JPC, Robertson RM (1986) Neuronal circuits: an evolutionary perspective. Science 233:849-853.

Elliott EJ, Muller KJ (1983a) Sprouting and regeneration of sensory axons after destruction of ensheathing glial cells in the leech central nervous system. J Neurosci 3:1994-2006.

Elliott EJ, Muller KJ (1983b) Accurate regeneration of an electrical synapse between two leech neurones after destruction of the ensheathing glial cell. J Physiol (Lond) 344:243-255.

Flaster MS, Zipser B (1987) The macroglial cells of the leech are molecularly heterogeneous. J Neurosci Res 17:176-183.
Flaster MS, Schley C, Zipser B (1983) Generating monoclonal antibodies against excised gel bands to correlate immunocytochemical and biochemical data. Brain Res 277:196-199.

Ghysen A (1978) Sensory neurons recognize defined pathways in Drosophila central nervous system. Nature 274:869-872.

Graziadei PP, Monti Graziadei GA (1986) Neuronal changes in the forebrain of mice following penetration by regenerating olfactory axons. J Comp Neurol 247:344-356.

Harris WA (1980) Regions of the brain influencing the projection of developing optic tracts in the salamander. J Comp Neurol 194:319333.

Hatten ME, Mason CA (1986) Neuron-astroglia interactions in vitro and in vivo. Trends Neurosci 9:168-174.

Heffner CD, Lumsden AGS, O'Leary DDM (1990) Target control of collateral extension and directional axon growth in the mammalian brain. Science 247:217-220.

Jacobs JR, Goodman CS (1989a) Embryonic development of axon pathways in the Drosophila CNS. I. A glial scaffold appears before the first growth cones. J Neurosci 9:2402-2411.

Jacobs JR, Goodman CS (1989b) Embryonic development of axon pathways in the Drosophila CNS. II. Behavior of pioneer growth cones. J Neurosci 9:2412-2422.

Katz MJ, Lasek RJ (1979) Eye transplanted to tadpole tails send axons in two spinal cord tracts. Science 199:202-204.

Letourneau P, Madsen AM, Palm SL, Furcht LT (1988) Immunoreactivity for laminin in the developing longitudinal pathway in the brain. Dev Biol 125:135-144.

Loer MC, Kristan WB (1989) Peripheral target choice by homologous neurons during embryogenesis of the medicinal leech. II. Innervation of ectopic reproductive tissue by non-reproductive Retzius cells. J Neurosci 9:528-538.

LoPresti V, Macagno ER, Levinthal C (1973) Structure and development of neuronal connections in isogenic organisms: cellular interactions in the development of the optic lamina in Daphnia. Proc Natl Acad Sci USA 70:433-437.

Lumsden AGS, Davies AM (1983) Earliest sensory nerve fibers are guided to the peripheral targets by attractants other than nerve growth factor. Nature 306:786-788.

Macagno ER, Gao W-Q, Baptista CA, Passani MB (1990) Competition or inhibition? Developmental strategies in the establishment of peripheral projections by leech neurons. J Neurobiol 21:107-119.

McKay RDG, Hockfield S, Johansen J, Thompson I, Frederiksen K (1984) Surface molecules identify groups of growing axons. Science 222:788-794.

Murphey RK, Bacon JP, Sakaguchi DS, Johnson SE (1983) Transplantation of cricket sensory neurons to ectopic locations: arborization and synaptic connections. J Neurosci 3:659-672.

Murphey RK, Bacon JP, Johnson SE (1985) Ectopic neurons and the organization of insect sensory neuropils. J Comp Physiol A 156:381389.

Palka J (1982) Genetic manipulation of sensory pathways in Drosophila. In: Neuronal development (Spitzer NC, ed), pp 121-170. New York: Plenum.

Palka J, Ghysen A (1982) Segments, compartments and axon paths in Drosophila. Trends Neurosci 5:382-386.

Patel NH, Snow PM, Goodman CS (1987) Characterization and cloning of fasciclin III: a glycoprotein expressed in a subset of neurons and axon pathways in Drosophila. Cell 48:975-988.

Peinado A (1988) Axon guidance and fasciculation in the peripheral nervous system of the leech Hirudo medicinalis. PhD thesis, Columbia University.

Peinado A, Zipser B, Macagno ER (1987a) Regeneration of afferent axons into discrete tracts within peripheral nerves in the leech. Brain Res 410:330-334.

Peinado A, Macagno ER, Zipser B (1987b) A group of related surface glycoproteins distinguish sets and subsets of sensory afferents in the leech nervous system. Brain Res 410:335-339.

Peinado A, Zipser B, Macagno ER (1990) Pathway selection by afferent projections in the leech Hirudo medicinalis. J Comp Neurol 301:232242.

Raper JA, Bastiani MJ, Goodman CS (1983) Pathfinding by neuronal growth cones in grasshopper embryos: selective fasciculation onto specific axonal pathways. J Neurosci 3:31-41.

Sawyer R (1986) Leech biology and behaviour. Oxford: Oxford UP. Schmid H, Gendre N, Stocker RF (1986) Surgical generation of su- 
pernumerary appendages for studying neuronal specificity in Drosophila melanogaster. Dev Biol 113:160-173.

Schneiderman A, Matsumoto SG, Hildebrand JG (1982) Trans-sexually grafted antennae influence development of sexually dimorphic neurons in moth brain. Nature 298:844-846.

Stent GS, Weisblat DA (1985) Cell lineage in the development of invertebrate nervous systems. Annu Rev Neurosci 8:45-70.

Stewart RR, Macagno ER, Zipser B (1985) The embryonic development of peripheral neurons in the body wall of the leech Haemopis marmorata. Brain Res 332:150-157.

Takagi S, Tsuji T, Amagai T, Takamatsu T, Fujisawa H (1987) Specific cell surface labels in the visual centers of Xenopus laevis tadpole identified using monoclonal antibodies. Dev Biol 122:90-100.

Tessier-Lavigne M, Plackzek M, Lumsden AGS, Dodd J, Jessell TM (1988) Chemotropic guidance of developing axons in the mammalian nervous system. Nature 336:775-778.

Tomaselli KJ, Neugebauer KM, Bixby JL, Lilien J, Reichardt LF (1988)
$\mathrm{N}$-cadherin and integrins: two receptor systems that mediate neuronal process outgrowth on astrocyte surfaces. Neuron 1:33-43.

Torrence SA, Stuart DK (1986) Gangliogenesis in leech embryos: migration of neural precursor cells. J Ncurosci 6:2736-2746.

Weisblat DA, Harper G, Stent GS, Sawyer RT (1980) Embryonic cell lineages in the nervous system of the glossiphoniid leech Helobdella triserialis. Dev Biol 76:58-78.

Yamamoto M, Boyer AM, Crandall JE, Edwards M, Tanaka J (1986) Distribution of stage-specific neurite associated proteins in the developing murine nervous system recognized by a monoclonal antibody. J Neurosci 6:3576-3594.

Zipser B, McKay RDG (1981) Monoclonal antibodies distinguish identifiable neurons in the leech. Nature 289:549-554.

Zipser B, Morell R, Bajt ML (1989) Defasciculation as a neuronal pathfinding strategy: involvement of a specific glycoprotein. Neuron $5: 621-630$. 